\title{
Ground Glass Opacity
}

National Cancer Institute

\section{Source}

National Cancer Institute. Ground Glass Opacity. NCI Thesaurus. Code C113718.

A CT scan finding in the lungs indicating the presence of hazy areas of attenuation in the air spaces. It is caused by interstitial fibrosis, alveolar collapse, edema, inflammation, or cancer with lepidic spread. 